\title{
EDS Windows and Plasma Cleaning: Characterization and Damage Mechanisms
}

\author{
Jens Rafaelsen ${ }^{1}$
}

1. EDAX Inc, A division of Ametek, Materials Analysis Division, 91 McKee Drive, Mahwah, NJ

The desire to reduce carbon contamination in the scanning electron microscope (SEM) chamber has been one of the driving factors in the shift from oil diffusion pumps to turbo pumps and the replacement of oil lubricated roughing pumps to dry pumps. A further step in reducing chamber contamination is the addition of a plasma cleaner to the microscope chamber. The cleaning process is based gas molecules (often atmospheric air) passing through a radio frequency (RF) cavity to generate radicals. The radicals enter the SEM chamber where they can react with various species of contaminants to bring them into gas phase and thus be pumped out by the vacuum system.

The removal of carbon contamination can have a significant impact on image quality especially when using low acceleration voltages $(<5 \mathrm{kV})$. The signals detected in Energy-dispersive X-ray Spectroscopy (EDS) can also reap notable benefits from the removal of surface contamination ${ }^{[1]}$. Especially in the energy range below $500 \mathrm{eV}$, the presence of a carbon layer can introduce a significant attenuation of the $\mathrm{x}$-ray signal as can be seen from the mass attenuation coefficient of carbon shown in Figure 1. However, attenuation of the generated signal is not the only challenge a micro-analyst faces when conducting EDS measurements with low acceleration voltages. Figure 2 shows how a significant part of the electron beam energy is deposited in a carbon over layer as the acceleration voltage decreases, resulting in significantly reduced X-ray generation in the substrate.

One problem with the combination of EDS and plasma cleaning is the question of window durability. Traditionally, EDS windows are made of a multilayer structure of polymer and aluminum with a new alternative being a multilayer structure of silicon nitride and aluminum. In this study we examine the damage mechanism to the polymer windows and the differences between silicon nitride and polymer windows. While both windows utilize a thin Al protective layer which also works as a light shield, the Al layer plays a vital part in reducing plasma cleaner damage to the polymer window as shown in Figure 3.

To characterize the efficiency of the plasma cleaning we measure the removal rate of carbon deposited on a Si substrate and the corresponding effect on an Al layer on a Si substrate. These findings are then applied to actual EDS modules with both polymer and silicon nitride windows. The damage effects are documented by EDS measurements and focused ion beam (FIB) preparation of the window structure as shown in Figure 4.

\section{References:}

[1] Improved Carbon Analysis with Evactron Plasma Cleaning. Rolland, P., Carlino, V. and Vane, R. . S02, s.1. : Microscopy Society of America, 2004, Microscopy and Microanalysis, Vol. 10, pp. 964-965x. [2] P. T. Törmä et al, IEEE TRANSACTIONS ON NUCLEAR SCIENCE, 61 (2014), pp. 695-699.

[3] Chantler, C.T. et al. "X-Ray Form Factor, Attenuation and Scattering Tables (version 2.1).". National Institute of Standards and Technology (NIST). Retrieved February 2016

[4] D. Drouin et al, SCANNING VOL. 29, 92-101 (2007) 


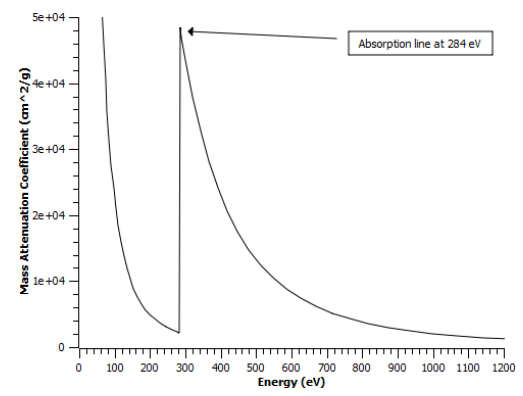

Figure 1. Mass attenuation coefficient of Carbon in the $0-1200 \mathrm{eV}$ range ${ }^{[3]}$.

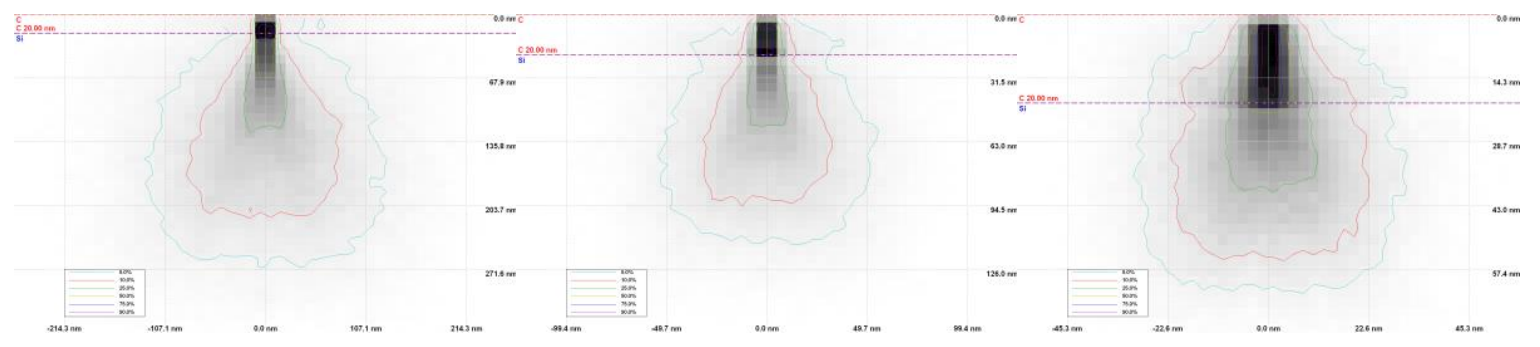

Figure 2. Simulation using the $\mathrm{CASINO}^{[4]}$ software of the absorbed energy by position at different electron energies in Si sample with $20 \mathrm{~nm} \mathrm{C}$ coating. (Left) $5 \mathrm{keV}$. (Center) 3keV. (Right) $2 \mathrm{keV}$.

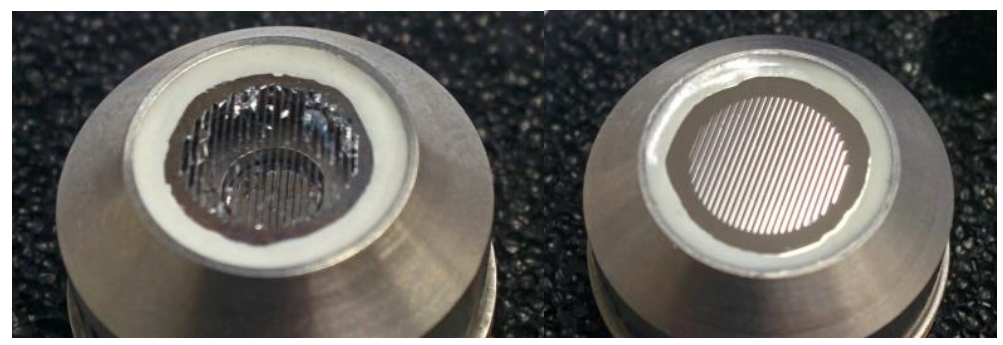

Figure 3. Optical photograph of two EDS detector modules with polymer windows. Both modules were exposed to oxygen radicals in a plasma asher chamber for 24 hours. The plasma source was running at $50 \mathrm{~W}$ at a pressure of $2 * 10^{-2}$ mbar. The module on the left had a damaged Al layer and suffered complete window failure while the module on the right shows no obvious signs of degradation.

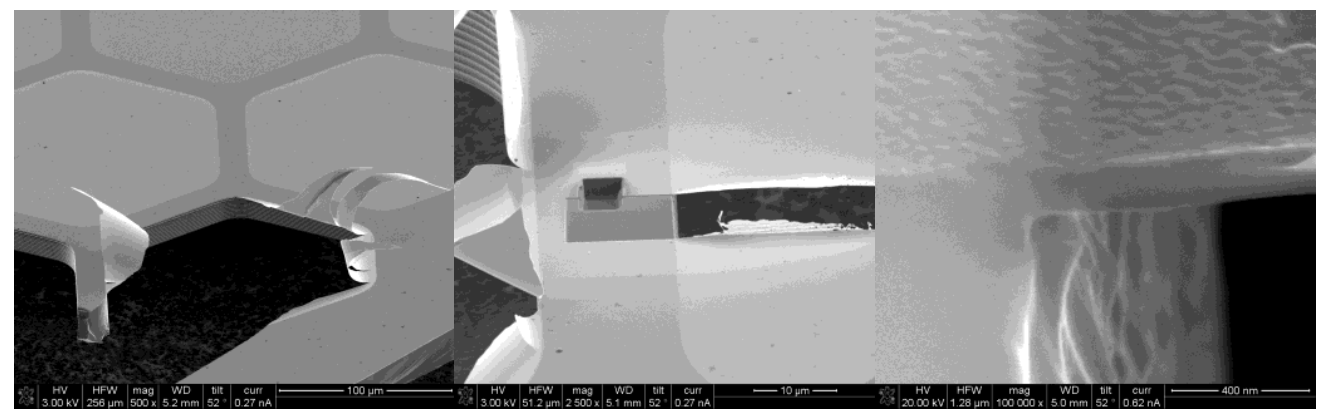

Figure 4. SEM images of silicon nitride window structure. (Left) The window has been removed from the detector module and the hexagonal support structure can be seen. (Center) FIB cut into the support structure and through the unsupported silicon nitride window. (Right) High magnification image of the silicon nitride structure with Al layer on top. 